\title{
Myasthenia Class III
}

National Cancer Institute

\section{Source}

National Cancer Institute. Myasthenia Class III. NCI Thesaurus. Code C112016.

Moderate weakness affecting non-ocular muscles. May also have ocular muscle weakness. 